LBL-25667

ESG Note-54

\title{
BEAM DYNAMICS ACTIVITIES AND PLANS AT LBL*
}

\author{
S. Chattopadhyay \\ Lawrence Berkeley Laboratory \\ University of California \\ Berkeley, CA 94720
}

July 1988

Contributed to the Beam Dynamics Newsletter of ICFA (International Committee for Future Accelerators).

* This work was supported by the Office of Energy Research, Office of Basic Energy Sciences, Department of Energy under Contract No. DE-AC03-76SF00098. 


\title{
BEAM DYNAMICS ACTIVITIES AND PLANS AT LBL
}

\author{
S. Chattopadhyay \\ Lawrence Berkeley Laboratory \\ University of California \\ Berkeley, CA 94720
}

On-going beam dynamics studies at LBL are performed in connection with the 1-2 GeV Advanced Light Source (ALS), the SSC, Collider Physics (Novel Power Sources) and Heavy Ion Fusion Accelerator Research (HIFAR). Exploratory activities include generic research on (a) highbrightness, low-emittance storage rings and linacs for the TeV-scale future linear colliders, $b \bar{b}$ facilities, etc., (b) beam dynamics and optics of incoherent and coherent undulator radiation, (c) Free Electron Laser Studies, (d) development of a high-brightness laser-driven RF photocathode electron source for the LBL-LLNL-SLAC collaboration on Relativistic Klystrons and (e) new methods of acceleration.

The major physics issues being studied in connection with the ALS are the effects of undulators and wigglers on storage ring beam dynamics (nonlinear stability and stable dynamic aperture), short bunch-length collective phenomena and multi-loop high-fidelity orbit and photon beam feedback for stability of the radiation source. In connection with the first issue, detailed nonlinear behavior of the electron beam in presence of insertion devices in an otherwise symmetric low-emittance lattice are being pursued and various compensation schemes are being investigated. We held a workshop on this specialized topic May 17-20, 1988 at LBL, where participants from international laboratories such as Sincrotrone Trieste, KEK, SLAC, BNL, ANL, etc., participated. Contributions will soon be published as proceedings to this workshop. Considerable insight into the physics of short bunches has been gained through a similar workshop last year on the "Impedance Beyond Cutoff" at LBL, where a realistic picture of the impedance spectrum at high frequencies corresponding to short bunch lengths emerged. The focus at the moment is on the nature of coherent instabilities potentially driven by this high-frequency impedance. Securing a stable photon beam against vibrations and other noise sources simultaneously at several beam lines in a synchrotron radiation source is nontrivial and requires multi-loop orbit feedback system with accompanying cross-talk. A possible control algorithm is under investigation. In other matters, experimental study of dynamic effects with undulators is being planned at BESSY and other laboratories; impedance measurement test facility, instrumentation and feedback, advanced 
accelerator control systems and optics of synchrotron radiation are being pursued actively. Possibilities of infra-red and XUV FEL's as upgrades to the ALS are also being considered.

Collaboration with LLNL and SLAC for the Relativistic Klystron is an ongoing activity. This promising power-source is being actively investigated both theoretically and experimentally with a goal towards a $1 \mathrm{GeV}$ test experiment in late 1989. In this connection, studies of a conceptual design of a bright injector for the test experiment has been undertaken at Berkeley. The Conceptual Design Report will be completed in October, 1988. The design utilizes a highbrightness laser-driven RF photocathode electron gun, similar to the LANL early experiments, together with achromatic magnetic bunching and transport systems and diagnostics. The design is performed with attention to possible use in an FEL as well.

Heteroenergetic collision optics for a possible $b \bar{b}$ facility based on $12 \mathrm{GeV}$ PEP and a $2 \mathrm{GeV}$ high-brightness ring has been obtained. The possible implications of collective effects is under study presently.

A small but core group of physicists are engaged in pursuing the frontier of knowledge in advanced and efficient methods in nonlinear storage ring dynamics. Extraction of precise nonlinear maps for a modular storage ring, perturbative \& nonperturbative analysis on these maps, stability issues, etc., are being pursued. Development of analytical and tracking tools is a major thrust of this activity.

In HIFAR, major attention is focused on two experiments, ILSE and MBE-4. A major cost saving in multiple beam ion induction linacs for fusion arises from the use of a large number of beams (approx. 100) at low energies which are combined into a smaller number after some acceleration. The study of the transverse emittance growth arising from the merging of 16 space charge dominated beams into 4 , and the design of the transport system to affect this merge are two of the principal tasks of the forthcoming ILSE (Induction Linac Systems Experiment). The beam dynamics from the time the beams are brought near each other and exposed to each others fields until the new distribution of particles reaches a stable configuration is being studied with particle simulation. The results are becoming understood in terms of a geometric dilution term, which is the same as for ordinary emittance dominated beam merging, and a space charge term which arises from transfer of electrostatic energy into thermal energy. In addition to these transverse beam dynamics studies, longitudinal beam dynamics is also of serious concern. Control of the lengths of the beam bunches during the acceleration process is one of the key beam dynamics issues of the 
multiple-beam induction linac as an accelerator-driver of heavy ions for inertial fusion. This physics is being studied experimentally in the MBE-4 experiment, that models the longitudinal dynamics of the first $100 \mathrm{MeV}$ of a driver. Measurements of the current amplification and of the behavior of transverse and longitudinal emittance of the four beams as functions of time are currently in progress. Control of the accelerating waveforms, within reasonable amplitude and time jitter tolerances, for proper bunch shaping and emittance is a crucial issue in this study.

In addition, considerable effort is dedicated to student training and Ph.D. thesis supervision in Accelerator Physics, in conjunction with the Berkeley campus. 